\title{
Analysis of separatrix plasma parameters using local and multi-machine databases
}

\author{
G.D. Porter ${ }^{\mathrm{a}, *}$, S. Davies ${ }^{\mathrm{b}}$, B. LaBombard ${ }^{\mathrm{c}}$, A. Loarte ${ }^{\mathrm{d}}$, K. McCormick ${ }^{\mathrm{e}}$, \\ R. Monk ${ }^{b}$, M. Shimada ${ }^{f}$, M. Sugihara ${ }^{g}$ \\ ${ }^{a}$ Lawrence Livermore National Laboratory, P.O. Box 85608, Livermore, CA 94551-9900, USA \\ b JET Joint Undertaking, Abingdon, Oxon, OX14 3EA, UK \\ c Plasma Fusion Center, Massachusetts Institute of Technology, Cambridge, MA 02139, USA \\ d The NET Team, Max-Planck-Institut für Plasmaphysik, D-85748 Garching, Germany \\ ${ }^{\mathrm{e}}$ Max-Planck-Institut für Plasmaphysik, EURATOM Assoc., D-85748 Garching, Germany \\ ${ }^{\mathrm{f}}$ JAERI, Naka Fusion Research Establishment, Ibaraki-ken 311-01, Japan \\ g ITER Joint Central Team, Joint Working Site, D-85748 Garching, Germany
}

\begin{abstract}
The characteristics of the plasma density and temperature at the edge of a diverted tokamak are presented. The analysis is done using the edge database formed by the ITER divertor database and modeling expert group. Analysis indicates the density profile is flatter with high density operation, i.e. the separatrix density is a larger fraction of the average density. This characteristic is found to be independent of plasma operation mode. We find the separatrix density in ITER will be approximately $6 \times 10^{19} \mathrm{~m}^{-3}$, based on scaling from the major diverted tokamaks. Scaling of the edge temperature is more problematic. A model which concludes the edge temperature is sensitive to details of the recycling process is presented. The temperature profile is analyzed to obtain the perpendicular thermal diffusivity. We find a diffusivity of $0.2 \mathrm{~m}^{2} / \mathrm{s}$ for ITER H-mode operation. The existing data, however, does not permit any size scaling of the diffusivity. (c) 1999 Elsevier Science B.V. All rights reserved.
\end{abstract}

Keywords: Database; ITER; Edge plasma

\section{Introduction}

Knowledge of the plasma parameters at the last closed flux surface (the separatrix) of diverted tokamaks is important for the design of divertors capable of removal of power and particles without deleterious effect on core confinement. The ITER design utilizes enhanced radiation in the divertor region to reduce the power load to the divertor [1]. This design is based in part on simulations with 2-D fluid plasma codes. The efficacy of the ITER design depends on the viability of the plasma

\footnotetext{
${ }^{*}$ Corresponding author. Tel.: +1-925 423 9391; fax: +1-925 424 6401; e-mail: porter2@llnl.gov.

${ }^{1}$ Work supported by the US DoE under Contract No. DEAC03-89ER51114 at GA and W-7405-ENG-48 at LLNL.
}

codes. The code simulations have been validated against data for a limited number of discharges in each of the world's major divertor tokamak devices, as reviewed by Loarte at this conference. A second technique which can be used to evaluate our understanding of the physics of the plasma scrape-off layer (SOL) and divertor is comparison with a broad range of experimental data. The ITER divertor modeling and database expert groups have assembled a scalar database of the edge plasma parameters for existing diverted tokamak devices as a means of enabling scaling studies of the SOL plasma. Data exist from ASDEX, ASDEX-Upgrade, C-MOD, COMPASS-D, DIII-D, JET, JFT-2M and JT-60U. We describe the scaling of plasma parameters at the outer midplane obtained from examination of this multi-machine database in this paper. The plasma parameters at the outer midplane are used as a boundary condition for 
all simulations of the SOL plasma and hence understanding of the expected value of these parameters is crucial for the successful design of the ITER divertor.

A major difficulty that we encounter in building a database of the plasma parameters at the separatrix is the determination of the exact location of the separatrix. The spatial location of the separatrix is determined by reconstruction of the magnetic geometry from measurements outside the plasma [2]. The uncertainty of the separatrix location can be large, on the order of $1 \mathrm{~cm}$. The effect of this uncertainty on the plasma parameters at the separatrix depends on the operating mode of the plasma. The radial scale length of the plasma density and temperature range from a few centimeters in Ohmic and L-mode operation to a few millimeters in H-mode. It has been shown that the inherent uncertainty in the separatrix location leads to unacceptably large uncertainty in the plasma parameters in H-mode plasmas, hence we must apply corrections for this uncertainty [3]. The techniques used for correcting the data include forcing pressure balance between the divertor and upstream (typically at the outer midplane) plasma; forcing consistency with a model of conduction limited parallel transport; and defining the separatrix to lie at one-half the electron temperature radial scale outside the maximum temperature gradient. The data presented in this paper include our best attempts at using these corrections. Unfortunately, we do not have corrections for all data from all devices.

\section{Scaling of separatrix density}

Typically the core plasma density is determined by externally applied neutral gas injection in Ohmic and Lmode operation. Improved particle confinement means the density rises above the desired density in H-mode, so neutral gas injection is usually set to zero and the plasma density is determined by sources from neutral beam injection and neutral recycling. The density obtained at the separatrix is expected to be determined by a balance between particle flow out of the core and ionization of neutrals arising from recycling of ions lost from the core plasma. The density at the separatrix is then expected to be related to some measure of the core density and the efficiency of ionization of the recycling neutrals on closed flux surfaces. We use the line averaged density obtained from interferometer measurements as a measure of the core density. The dependence of the separatrix density on the line averaged core density is shown in Fig. 1. There is remarkably little variation in the relationship between the core and separatrix density among the different devices in spite of very different divertor geometries. It should be noted, however, that there is at least a factor of 2 variation in the separatrix density at a given line averaged density. The insensitivity

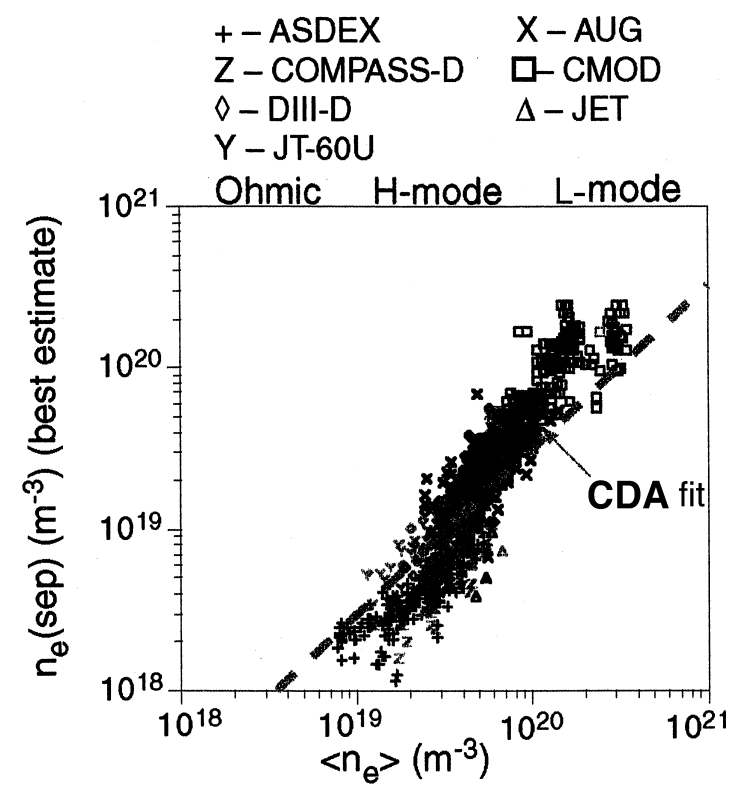

Fig. 1. The dependence of the separatrix density on the lineaveraged core density.

to device indicates the dependence of the separatrix density on divertor geometry is within the statistical variation. The density profile is seen to be flatter, i.e. higher separatrix density relative to line averaged density, for high density operation. The separatrix density assumed during the CDA phase of ITER, 30\% of the average core density, is shown in the figure for reference. Note that the separatrix density obtained in H-mode does not differ substantially from that obtained in Ohmic or L-mode, relative to the average core density. The improved particle confinement time obtained in $\mathrm{H}$ mode drives the device to higher density and all high density operation obtains relatively flat density profiles $\left[0.5 \leqslant n_{\mathrm{e}}(\mathrm{sep}) /\left\langle n_{\mathrm{e}}\right\rangle \geqslant 1.0\right]$. The data shown in Fig. 1 indicates the separatrix density of ITER would be about $6 \times 10^{19} \mathrm{~m}^{-3}$, for an average core density of $10^{20} \mathrm{~m}^{-3}$. The statistical variation, however, suggests the ITER separatrix density might lie anywhere between $3 \times 10^{19}$ and $10^{20} \mathrm{~m}^{-3}$.

An alternate technique which can be used to scale the separatrix density expected in ITER would be to seek the best empirical fit to the data in the scalar database. A simple log-linear fit of the separatrix density to the average core density suggests $n_{\mathrm{e}}(\mathrm{sep}) \propto\left\langle n_{\mathrm{e}}\right\rangle^{1.6}$. However, there are other hidden variables which cannot be ignored. For example the highest density data in the database is obtained from C-MOD which also operates at the highest magnetic fields. Hence there may be other device dependencies which determine the separatrix density. The Ohmic and L-mode data is best fit with the function 
$n_{\mathrm{e}}(\mathrm{sep})=0.00236\left\langle n_{\mathrm{e}}\right\rangle^{1.08} \kappa^{1.11} B_{\mathrm{T}}^{0.78}$

where $\kappa$ is the plasma elongation and $B_{\mathrm{T}}$ is the toroidal magnetic field in Tesla. Both the line averaged density and the separatrix density are expressed in units of $\mathrm{m}^{-3}$. The separatrix density in H-mode is consistent with this empirical scaling, but there is a degradation of core energy transport with flattening of the density profile. We have not included $\mathrm{H}$-mode data in our fit because this dependence on confinement is not included. Somewhat poorer fits can be obtained using the heating power as a variable, but the dependence on power is very weak. The dependence on a geometry parameter such as the plasma elongation may indicate the contribution of recycling to determination of the separatrix density. However, the physical mechanism by which the particular parameters enter Eq. (1) is not obvious. The edge density predicted by Eq. (1) for ITER is about 6.2 $( \pm 1.4) \times 10^{19} \mathrm{~m}^{-3}$, similar to that obtained directly from Fig. 1. The edge density expected in ITER from either of these techniques is somewhat higher than that used during the CDA, making design of the divertor easier.

\section{Examination of separatrix temperature and perpendic- ular transport coefficients}

A model discussed in this section suggests that the separatrix temperature can be sensitive to the recycling process, which may explain the observed scatter in the deduced radial energy transport coefficients. We can divide the tokamak plasma into three regions; the core plasma, edge plasma and SOL. The edge plasma is the region in which the $\mathrm{H}$-mode thermal barrier is established and typically lies between normalized poloidal flux surfaces of 0.9 and 1.0 , i.e. out to the separatrix. The edge plasma is heated by radial transport from the core plasma. Cooling mechanisms for the edge include radial thermal conduction to the SOL, impurity radiation, convective losses to the SOL by large particle fluxes and parallel losses in the SOL. Previous scaling for the separatrix temperature were based on balancing the perpendicular conductive power flow across the separatrix with parallel conduction to the plate [4]. We propose here that the convective power loss is important, especially in H-mode. The particle flux across the separatrix is determined by the total ionization rate in the core and includes ions which are created by ionization of recycling neutrals in the edge region. Ions which originate from this ionization process diffuse rapidly across the steep gradient at the separatrix, hence they do not contribute to the separatrix density. However, the thermal equilibration time between the cold electrons and ions introduced by the ionization process and the background plasma is rapid. Hence radial convective power loss can be significant, cooling the plasma in the edge. We expect, therefore, to find the separatrix temperature to be sensitive to recycling and thus to details of the divertor geometry, wall conditioning, pumping, etc.

This hypothesis can be examined by determining the perpendicular thermal and particle diffusivities from the measured radial density and temperature profiles. Assuming a single species model, the power and particle flow can be written

$$
\begin{aligned}
P_{\perp} & =-2 \chi\left(\frac{\partial(n T)}{\partial r}+(\alpha-1) T \frac{\partial n}{\partial r}\right) A_{\text {sep }}, \\
I_{\perp} & =-D A_{\text {sep }} \frac{\partial n}{\partial r} \\
& =\frac{\alpha P_{\perp}}{5} \frac{\partial n / \partial r}{(\partial(n T) / \partial r+(\alpha-1) \partial n / \partial r)},
\end{aligned}
$$

where $D$ is the radial particle diffusivity, $\chi$ is the radial thermal diffusivity, $\alpha=2.5 \mathrm{D} / \chi, P_{\perp}$ is the total power flowing radially and $A_{\text {sep }}$ is the surface area of the separatrix. The expression for the perpendicular current is derived assuming particle flow is purely diffusive, i.e., pinch effects are small. The parameter $\alpha$ has been estimated to be about 0.6 in DIII-D by comparing the radial particle flux determined from Eq. (2) just prior to the Lto $\mathrm{H}$-mode transition with the rate of rise of the density after the transition [5]. We do not have estimates of the value of $\alpha$ for other devices, so we assume 0.6 for all devices.

Determination of the radial diffusivities requires knowledge of not only the density and temperature at the separatrix, but also their gradients. Hence it is particularly sensitive to uncertainties in the separatrix position. Although we have attempted to correct for uncertainties from the magnetic reconstruction, we cannot be certain this has been done correctly. In fact only $\mathrm{H}$-mode data from DIII-D has been corrected in all aspects. None the less, it is instructive to consider the behavior of the diffusivity we find by evaluating Eq. (2). We approximate $P_{\perp}$ as the difference between the heating power and the power radiated in the core. The resulting thermal diffusivity is shown in Fig. 2. The thermal diffusivity increases with surface heating power. The diffusivity will be linear with heating power for constant gradients, so the results shown in Fig. 2 indicate the gradients are only weakly dependent on power. The diffusivity for Ohmic and L-mode are up to an order of magnitude higher than those for H-mode. One possible source of the large scatter seen in Fig. 2 may be uncertainties in the location of the separatrix.

The effect of convective power loss associated with particle recycling can be inferred from the data shown in Fig. 3, which shows the variation of the separatrix electron temperature with the radial particle flux obtained from Eq. (2). The particle fluxes inferred from the data are on the order of a factor of 10 higher than the particle input from neutral beam heating. This suggests 


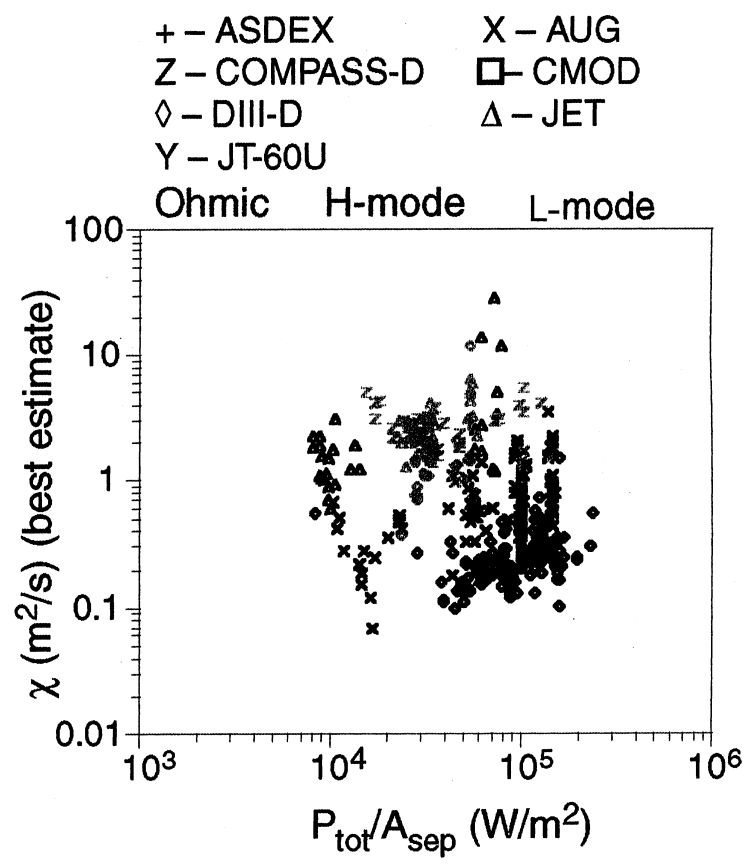

Fig. 2. Variation of the radial thermal diffusivity at the separatrix with the surface radial power density.

the radial particle flux is dominated by ionization of recycling neutrals. The separatrix electron temperatures obtained in $\mathrm{H}$-mode are higher than those in Ohmic and L-mode and the temperature decreases as the radial

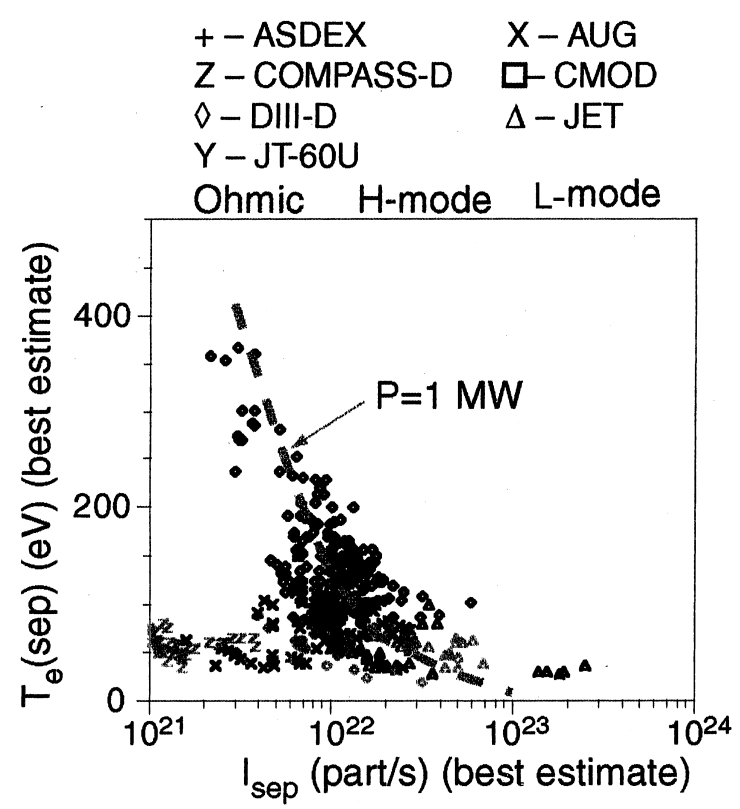

Fig. 3. Dependence of the separatrix electron temperature on the radial particle flux across the separatrix. particle flux increases. We show the $1 \mathrm{MW}$ convective power loss obtained from $P_{\text {conv }}=2.5 I_{\text {sep }}\left(2 T_{\mathrm{e}}\right)$ in the figure. We see that the convective power loss becomes significant at the high edge temperatures obtained in $\mathrm{H}$ mode. These results indicate that the separatrix temperature expected for ITER will be dependent on details of the recycling process, hence will depend on the divertor design.

\section{Discussion}

We have shown initial scaling dependencies for the plasma parameters at the outer midplane. The data are obtained from a variety of operating divertor tokamaks and has been assembled by the ITER Divertor modeling and database expert group. We find the scaling of the separatrix density does not vary much between different devices. The density profile varies with plasma density, becoming flatter at higher density. The scaling of the separatrix density is similar in H-mode, Ohmic and Lmode plasmas. The plasma density is higher in H-mode, hence we find they have flatter density profiles. We expect a separatrix density of about $6( \pm 1.4) \times 10^{19} \mathrm{~m}^{-3}$ for ITER.

We have argued that the separatrix temperature may be more sensitive to details of the neutral recycling process. Convective power losses across the separatrix which arise because of ionization of the recycling neutrals are important power loss mechanisms in the edge of $\mathrm{H}$-mode plasmas. Hence the separatrix temperature at the outer midplane depends rather sensitively on details of the baffling of the divertor region. The separatrix temperature expected in ITER will therefore depend on details of the divertor design. We cannot obtain a scaling law from the existing data, but must rely on 2-D code simulations which include realistic models for neutral recycling. The separatrix temperature obtained in these simulations will be determined by specifying the power across the separatrix and the radial thermal and particle diffusivities. The diffusivities expected for ITER can be estimated from data such as that shown in Fig. 2. Assuming a surface area of $1200 \mathrm{~m}^{2}$ and radial power of $100 \mathrm{MW}$, the ITER H-mode thermal diffusivity would be approximately $0.2 \mathrm{~m}^{2} / \mathrm{s}$, similar to that found in DIII-D and ASDEX-Upgrade. Note, however, there is no size scaling since the data are all from similar sized devices. Hence any estimate of the thermal diffusivity in ITER should be considered to be only an initial approximation.

The observation that the separatrix temperature is affected by recycling phenomena emphasizes the coupling between the physics of the edge/SOL plasma and the physics of the core plasma. It has been observed [5] that the $\mathrm{H}$-mode core energy confinement time increases as the temperature in the edge region increases. The temperature at the top of the H-mode pedestal increases 
linearly with the separatrix temperature so it is impossible to determine which region is most critical for core confinement. The results described here suggest one can optimize the energy confinement in a tokamak by careful control of neutral recycling. This is consistent with the observation that many, if not all, of the improved energy confinement modes have been obtained after improvements in wall conditioning technology. We expect wall conditioning to affect the recycling physics and hence the edge temperature. Improved baffling of the divertor region, together with neutral pumping in the divertor, will also reduce the convective power loss from the edge and hence can be expected to improve the core energy confinement. This coupling between the physics of the edge/SOL and that of the core suggests the value of expanding the 2-D models used in divertor simulations to include the physics of the core transport. We can only include consistent boundary conditions between the core and SOL regions by using such coupled core/SOL models.

\section{Acknowledgements}

The authors wish to acknowledge fruitful discussion with members of ITER Divertor Modeling and Data- base Expert Group and ITER Divertor Physics Expert Group. The databases used in the present analysis were supplied by tokamak teams all over the world. Computational support by the IPP Information Department and the computer supporting staff of ITER Garching Joint Work Site is also acknowledged. The authors wish to express their sincere thanks to all members of these teams.

\section{References}

[1] G. Janeschitz, K. Borrass, G. Federici, Y. Igitkhanov et al., J. Nucl. Mater. 220-222 (1995) 73.

[2] L.L. Lao, H.S. John, R.D. Stambaugh, A.G. Kellman et al., Nucl. Fusion 25 (1985) 1611.

[3] G.D. Porter, J. Moller, M. Brown, C. Lasnier et al., Phys. Plasmas 5 (1998) 1410.

[4] S.I. Itoh, K. Itoh, A. Fukuyama, J. Nucl. Mater. 220-222 (1995) 117.

[5] G.D. Porter and the DIII-D team, The role of radial particle flow on power balance in DIII-D, submitted to Phys. Plasmas. 